\title{
Basics on 3D Ultrasound
}

\author{
Lúcio Borges Cruz \\ Department of Interventional Ultrasound, PROMEDICA GROUP/PA—Salvador, Bahia, Brazil
}

Correspondence: Rua João da Silva Campos, 1394, Itaigara - Salvador, Bahia, Brazil, CEP 41-815-200

Phone: 55-71-99647882, e-mail: lucio_borges@hotmail.com

\begin{abstract}
In the last few years, technology has progressed to make ultrasound imaging a viable tool. Following Food and Drug Administration (FDA) approval of 3-dimensional ultrasound (3DUS) in November 1997, interest has increased to get more benefits from this new advance.

Scientific advances in imaging processing in recent years have taken ultrasound into the next generation. Until recently, processing speeds were still too slow to offer real time benefit to 3D ultrasound systems. New developments with motion estimation and imaging registration techniques can allow $3 \mathrm{D}$ data acquisition to be accomplished without the need for position sensing devices.

The purpose of this article is to review the basics on 3DUS, 3DUS the method and its mains advantages, limitations as well as to mention some frequent pitfalls. It is also aimed to remark important points in pre- and postprocessing.
\end{abstract}

Keywords: Basics, principles, 3D ultrasound, and technique.

\section{INTRODUCTION}

Technology has definitely progressed to make ultrasound imaging a viable tool where two dimensional ultrasound (2D-US) has become a widely used and accepted diagnostic one.

Ultrasound is now in the next generation due to the scientific advances in imaging processing. In order to form an impression of the 3D anatomy and pathology, many 2D images must be mentally integrated, pontentially resulting in a time consuming, inefficient and variable process. In November 1997, the Food and Drug Administration (FDA) approved the clinical use of 3dimensional ultrasound (3DUS) and interests have been increased to get more benefits from this new advance (Fig. 1).

The advent of 3D and 4D ultrasound allowed us into the mechanics thinking of a sonologist by acquiring the volume data and allowing reconstruction of images in different planes on the ultrasound machine or in the workstation. 4D ultrasound is also known as "Real-time 3D ultrasound". Here the processing powers of the computers has directly increased the capacity of the machine and we can acquire and display the 3D datasets with their multiplanar reformations and renderings in real time, as we scan the patient. Beside other, this has many benefits in obstetrical scanning where it is critical to analyze anatomy as it

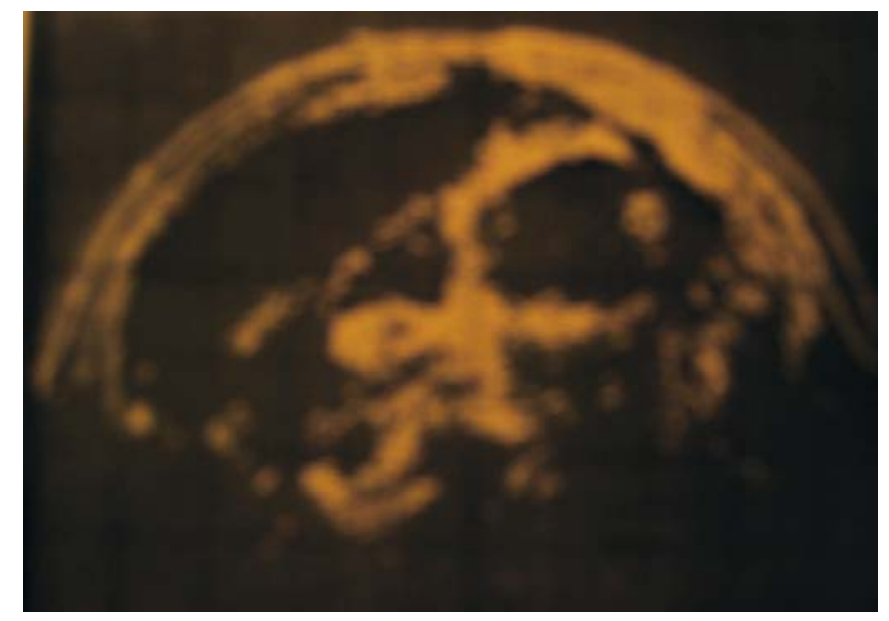

Fig. 1: US image aquired in 1974. An 18-weeks fetus

continues its physiological movements and allows the development of fetal psychology. ${ }^{1,2}$

Quantitative estimation of volume is usually performed from 2D images which may lead to inaccurate and variable results. It is difficult to localize the 2D image plane and to find the same location again at a later time, it makes 2D ultrasound ill-suited for monitoring therapy in real-time or for prospective studies. No information is lost during the 3D reconstruction and a variety of rendering techniques are possible with the inconvenience of large data files generation. Three perpendicular planes are displayed simultaneously and can be rotated and translated in order to obtain accurate sections and suitable views needed for diagnosis and geometric measurements. These imaging modes are innovative features which have to be evaluated for clinical applicability and usefulness. Digital documentation of whole volumes enables full evaluation without loss of information at a later point. 3D technology provides an enormous number of technical options which have to be evaluated so we can extract the most from the tool. ${ }^{3}$

The new routine of working in a workstation manipulating these data in such format can be a problem to sonologists. The problem is that we are not used to it, what can be the first barrier to be broken. It takes some understanding of the basics of volume acquisition and manipulation to understand the 
dynamics of reconstruction. It is perhaps easier for radiologists who have dealt with axial imaging in CT and multiplanar imaging in MRI since they are used to sectional imaging. While the learning curve to have the acquisition of the volumes is short, the ability to render the volume using different algorithms seems a little more complicated. The basic steps are simple, and a logical approach to 3D and 4D scanning can help in its complete comprehension, and even makes it easier to scan in many instances. ${ }^{4}$

\section{OVERVIEW}

Before starting the volume acquisition, a few things need to the set and adjusted. It is essential to have a good B mode image, having the region of interest well-shown in the screen (Fig. 2). The angle of the acquired volume must be adjusted. This is the point where you put limits to the aimed area so the volumetric probe can be placed and scan as such. The quality of acquisition, directely related to time of acquisition, must be set up. A higher quality needs more time to be captured. Of course we should define if we want 3D or 4D and the primary plane of acquisitionlongitudinal or transverse. ${ }^{5,6}$

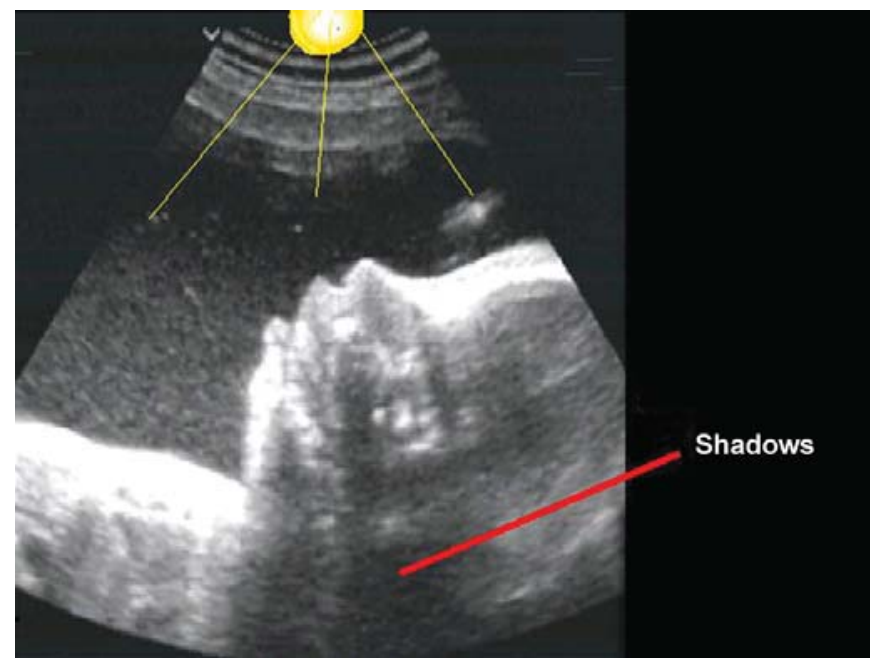

Fig. 2: Best 2D image for a 3D aquisition

In order to understand the three-dimensional imaging operations we may classify them under four basic headings: preprocessing, visualization, manipulation, and analysis. Preprocessing operations (volume of interest, filtering, registration, segmentation) are aimed at extracting or improving the extraction of object information in given images. Defining the object system to create a geometric model of the objects under investigation. ${ }^{7}$

Visualization operations facilitate seeing and comprehending objects in their full dimensionality and may be either scenebased or object-based. Viewing and comprehending the object system. Manipulation may be either rigid or deformable and allows alteration of object structures and of relationships between objects. Analysis operations, like visualization operations, may be either scene-based or object-based and deal with methods of quantifying object information. There are many challenges involving matters of precision, accuracy, and efficiency in 3D imaging. Quantifying information about object system.

\section{BASICS ON 3DUS}

Among the basic principles, we have the region of interest, that should be as small as possible, unless you want to look at a very large anatomy correlation. The angle of acquisition defines the swing/sweep of the probe and a wide angle will take longer acquisition time usually. The quality of the acquisition can be adjusted - the better the quality selected the longer is the acquisition time. The reason is simple - a better quality usually needs more image lines in the acquisition plane and more slices in the volume. For instance if you are scanning an active baby you will have to scan at lower quality to complete your acquisition faster, compromising the least the resolution of your data. Artifacts and noises can be pitfalls for the sonologists (Figs $3 \mathrm{~A}$ and $\mathrm{B}){ }^{8}$

It is important to decide, before you start the acquisition, whether you wish to have a 3D scan and a workstation afterwards or a 4DUS, with live real time display of all planes and rendering. A very thin volume 4D scan with some minor differences in mechanics of acquisition in the daily practice is called VCI (Volume Contrast Imaging) that can also be preselected in order to get the desired plane in real time while it increases the inherent contrast in the image and delineates borders in a better manner. $^{7}$

The available tools help the better imaging quality and consequently the interpretation of the data is warranted at this point. These are the tools that can help in understanding and improving the data. Translation is the property of leafing through the $2 \mathrm{D}$ planes in a volume and rotation is the way of turning the planes to improve orientation up down, right left. In order to rotate and translate the planes, we do need a point of correlation where its used a yellow dot to spot a lesion or pathology in all the corresponding planes (Figs $4 \mathrm{~A}$ and $\mathrm{B}){ }^{7}$

After we succeed in having the object, we can improve the image quality, color and contrast by using the rendering direction, using manners available to look at the rendered volume from one of the sides and rendering algorithms where we should be familiar with these issues such as: Surface texture, maximum, minimum modes, etc. It is important to mix the two modes chosen in a acceptable manner.

Two types of mechanical probes are available. The first one is a transducer with a rotating mechanical system that allows the acquisition of multiple images for their subsequent processing by a computer. This system is particularly useful 

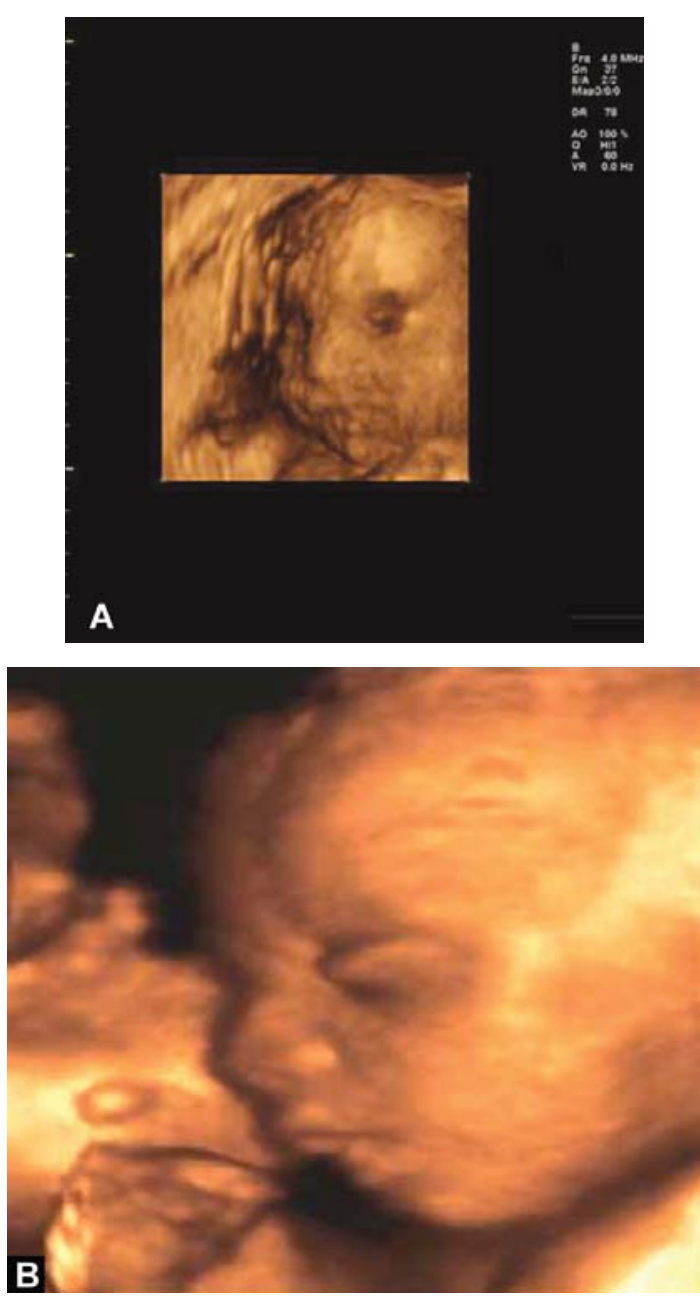

Figs 3 A and B: (A) Image of a 20-weeks moving fetus obtained in high velocity mode. Properly used for 4D. (B) Imaging in low velocity - good for 3D static pictures
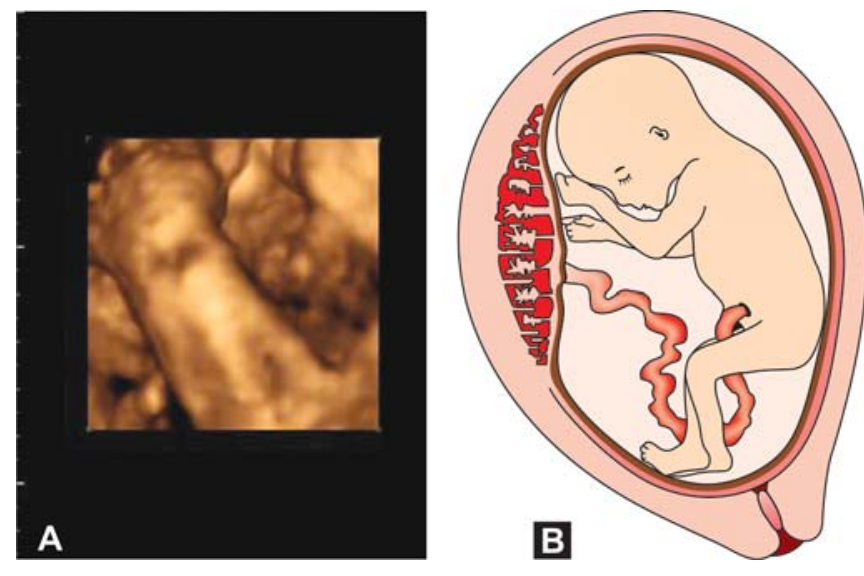

Figs 4A and B: No available angle due to the fetal position for small anatomic areas. The other is a more advanced mechanical system, capturing images from a truncated cone with its vertex located at the transducer. ${ }^{9}$

\section{The First Step-Acquiring}

In order to have the best acquisition of the cube, once the automatic scanning is initiated the hand must remain very still and the patient should be asked to hold breath briefly. This helps in reducing the breathing artifacts and gives better results.

Surface extraction from ultrasound data is challenging for a number of reasons including noise and artifacts in the images and nonuniform data sampling. Information is lost; and identifying boundaries is time-consuming resulting in susceptibility to inaccuracy and variability. Some artifacts can be created in presence of cardiac pulsation, breathing motion, improper calibration.

\section{The Second Step-Optimization}

Whenever we have the cube with the information needed inside, we should be skilled enough to get the maximal information available inside. The secrets is to know how to use the rendering algorithms and other tools to have various reconstructions. The use of gray maps and contrast adjustments with color available to apply and see if they can increase the yield of information. Almost every system allows two rendering modes with types and the percentages used in the mixture of the modes in the user's choice. ${ }^{10}$

The basic rendering algorithms are: Surface smooth and texture, transparency maximum and minimum, $\mathrm{X}$-ray mode and gradient light/light mode. It is mixing and matching any of the two algorithms, and then deciding the percentage of mixes that we get the best image. It is like mixing colors, textures, brightness or transparency until you have the best picture to illustrate the situation and make it understandable to physicians and patients. These various permutations and combinations can initially be experimented with and then you can find your comfort levels with regards to the rendering mixes you wish to use. Some general rules are present that can be used for as initial guidelinesminimum transparency for cystic structures, maximum for bony renderings, surface texturing for soft tissues, etc. ${ }^{10}$

What is volume rendering? Volume is a three-dimensional array of voxels. Just the same way an image is a 2D array of pixels. Voxel is the basic element of the volume. Typical volume size may be 1283 voxels, but any other size is acceptable. Volume rendering means rendering the voxel-based data into viewable 2D image. Types of rendering modes: Surface texture rendering, surface smooth rendering, light mode, X-ray rendering, max transparency rendering, min transparency rendering (Fig. 5).

Additionally you have three more tools to help in fine-tuning of your volume data and extracting more information: Threshold, magic cut and vocal-volume calculation. ${ }^{10-12}$ 


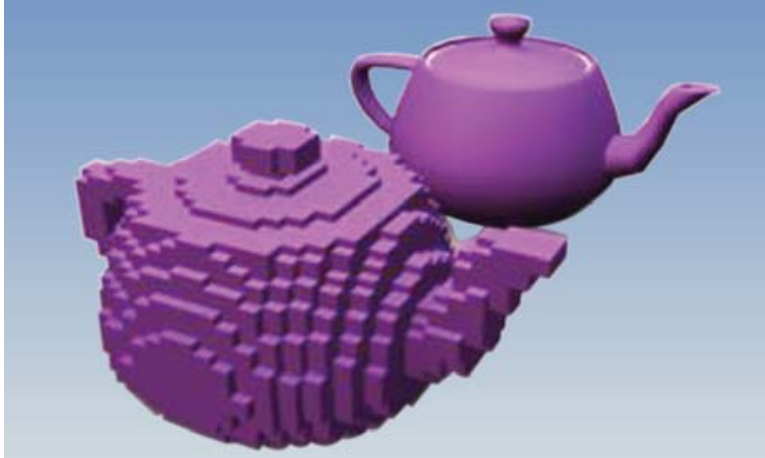

Fig. 5: Representative voxel array

\section{The Third Step-Navigating}

Tridimensional image reconstruction can be more complicated then the other steps, as slices are manually rather than automatically obtained, when using conventional 2D ultrasounds. Nevertheless the image acquisition depends on the explorer's skills. On the other hand, the number of slices that may be acquired is in numerous with no established beginning or end, as one more slice may always be obtained (Figs $6 \mathrm{~A}$ and B). ${ }^{8}$


Figs $6 \mathrm{~A}$ and $\mathrm{B}$ : Volume aquisition
Whenever it is time to start working with translation and rotation in order to exhibit the data, a reference point is needed, since the planes are exhibited in 2D. Usually a colored dot is used, which is seen in all planes, placed in such way that correlates the point of focus in all the planes. If you move this point and place it on a structure or a lesion in one plane, then the point automatically moves and finds the corresponding structure or lesion location in the other two planes. It is the same as seeing one voxel from different directions and is very important for localization (Fig. 7). ${ }^{7}$



Fig. 7: Yellow dots-reference points on each plane

\section{The Fourth Step-Analyzing}

This is the moment where you are analyzing the information we have acquired into a proper and coherent understanding. It is may be the most important step during the use of the 3D tool. It is time for us to realize whether we made a good job and to be sure that the information will fulfill our needs. Finally, it helps in choosing the right image or reconstruction to display, store or record the data.

\section{CONCLUSION}

We already know the importance of the clinical use of 2D ultrasound as a diagnostic tool. Due to the development of 
image processing, 3D and 4D ultrasound has become viable and available. Many benefits can be extracted from this new technique which has a short learning curve and allows acquisition and storage of data from the aimed area for future comparisons and follow ups. In order to succeed with the method, the sonologists shall go beyond scanning. It is important to learn to deal with the acquired data, postprocessing and visualization, using most of the devices displayed in the machines and workstations. 3D and 4D ultrasound has became a well-established technique, combining the acessibility of ultrasound with the possibility of reconstruction and assessment of the acquired data like is done in CT and MRI in nowadays.

\section{Conflict of Interest}

The author informs that there is no conflict of interest.

\section{ACKNOWLEDGMENT}

The author is thankful to Marco Antonio Guedes MD, on the contribution of the manuscript formation.

\section{REFERENCES}

1. Kurjak A, Azumendi G, Andonotopo W. Three- and fourdimensional ultrasonography for the structural and functional evaluation of the fetal face. AJOG 2007; 8(5): 174-87.
2. Udupa JK. Three-dimensional visualization and analysis methodologies: A current perspective. Radiographics 1999;19(3):783-806.

3. Steiner H, Staudach A, Spitzer D, Schaffer H. Diagnostic techniques: 3D ultrasound in obstetrics and gynaecology: Technique, possibilities and limitations. Human Reproduction 1994;9(9):1773-78.

4. Yen JT, Smith SW. Real-time rectilinear volumetric imaging using a periodic array. Ultrasound Med Biol 2002; 28(7):923-31.

5. Nelson TR, Downey DB, Pretorius DH, et al. Three-dimensional ultrasound in the evaluation of fetal anomalies. Lippincott Williams \& Wilkins, 1999.

6. Khurana A, Dahiya N. 3D and 4D Ultrasound: A text and atlas. Anshan, UK 2004.

7. Nirvikar Dahiya MD. The basics of 3D/4D ultrasound. GE Health Care Department brochure.

8. Rankin RN, Fenster A, Downey DB, Munk PL, Levin MF, Vellet AD. Three-dimensional sonographic reconstruction: Techniques and diagnostic applications. AJR 1993;161:695-702.

9. Candiani F. The latest in ultrasound: Three dimensional imaging Eur J Radiology 1998;27(supp 12):179-82.

10. Sakas G, Shirley P, Müller S. "Photorealistic Rendering Techniques. Springer-Verlag http://www.cs.wpi.edu/ matt/ courses/cs563/talks/powwie/p1/ray-cast.htm.

11. Watt $\mathrm{L}$, Watt $\mathrm{M}$. Advanced animation and rendering techniques Theory and Practice. Addison-Wesley, Reading, Massachusetts, 1992.

12. Levoy M. A hybrid ray tracer for rendering polygon and volume data, IEEE. Computer Graphics and Applications, 1990:33-40. 